\title{
Symptom Burden, Perceived Control, and Quality of Life Among Patients Living With Multiple Myeloma
}

\author{
Alexandra K. Zaleta, PhD'; Melissa F. Miller, PhD, MPH¹; Julie S. Olson, $\mathrm{PhD}^{1}$; Eva Y.N. Yuen, $\mathrm{PhD}^{1}$; \\ Thomas W. LeBlanc, MD, MA, MHS',3; Craig E. Cole, MD; Shauna McManus, BS ${ }^{1}$; and Joanne S. Buzaglo, PhD 1,5
}

\begin{abstract}
Background: New therapies for multiple myeloma (MM) have improved survival rates but often expose patients to heightened toxicities and prolonged treatment, leading to increasing complications and side effects. We evaluated the association between symptom burden, perceived control over illness, and quality of life (QoL) among a national sample of patients with MM. Methods: For this observational, cross-sectional study, we used data from the Cancer Experience Registry research initiative to examine symptom- and functioningrelated concerns among 289 patients with MM across the illness trajectory. We applied hierarchical multiple linear regression analyses to explore associations between symptom burden and perceived control over illness with QoL indicators: depression, anxiety, and social satisfaction. Results: In our sample, $73 \%$ of participants with $\mathrm{MM}$ reported currently receiving treatment; $39 \%$ experienced relapse; $56 \%$ received 1 to 2 autologous transplants, $10 \%$ received $\geq 3$ autologous transplants, and $4 \%$ received allogeneic and autologous transplants; $30 \%$ had not received a stem cell transplant. Average time since diagnosis was 4.4 years. The most highly endorsed concerns included eating and nutrition (61\%), physical activity (59\%), moving around (56\%), fatigue (55\%), pain (52\%), and sleep (46\%). Only $27 \%$ believed they had control over their disease, whereas $48 \%$ perceived having control over the physical side effects of MM. Approximately one-third of the variance in anxiety and depression and nearly two-thirds of variance in social satisfaction were explained by sociodemographic, clinical, and symptom burden variables. Perceived control over illness significantly predicted depression and anxiety, but not social satisfaction. Our results highlight substantial concern among patients with $\mathrm{MM}$ about physical symptoms and function. Additionally, greater symptom burden significantly accounted for poorer QoL, and lower perceived control over illness was linked to depression and anxiety. Conclusions: Patients with $\mathrm{MM}$ and survivors experience substantive long-term $\mathrm{QoL}$ issues. Together, these findings point to the critical need for comprehensive symptom management, integrated palliative care, and enhancement of social and emotional support for individuals with MM.
\end{abstract}

J Natl Compr Canc Netw 2020;18(8):1087-1095 doi: $10.6004 /$ jnccn.2020.7561

\footnotetext{
${ }^{1}$ Cancer Support Community, Research and Training Institute, Philadelphia, Pennsylvania; ${ }^{2}$ Duke Cancer Institute, and ${ }^{3}$ Division of Hematologic Malignancies and Cellular Therapy, Duke University School of Medicine, Durham, North Carolina; ${ }^{4}$ College of Human Medicine, Michigan State University, East Lansing, Michigan; and ${ }^{5}$ Now with Concerto HealthAl, Boston, Massachusetts.
}

\section{Background}

Multiple myeloma (MM), a plasma cell malignancy, is the second most common hematologic cancer in the United States, with an estimated 32,110 new cases occurring in 2019, and an estimated 12,960 deaths. ${ }^{1}$ Advances in treatment using autologous stem cell transplantation and novel therapies have resulted in significantly improved survival rates in the past 15 years. ${ }^{2-5}$ However, despite improved treatment, $\mathrm{MM}$ is incurable and progressive, and as such, the overarching aim of treatment-often interspersed with durations of remission with minimal or maintenance treatment ${ }^{6}$-is to control the disease and prolong survival. Although people with MM are living longer, they are doing so with increasing complications of the disease and cumulative side effects from prolonged treatment. ${ }^{7-9}$ Characteristic features of MM include destructive bone disease, bacterial infections, impaired renal function, anemia, and hypercalcemia. ${ }^{10}$ As a result, patients often experience significant adverse effects on quality of life (QoL), including fatigue, pain, breathlessness, muscle weakness, and peripheral neuropathy, ${ }^{11,12}$ which can negatively impact work, social, and familial roles. ${ }^{13}$ Research has shown patients with MM experience greater symptom burden and poorer QoL compared with those with other hematologic cancers. ${ }^{14}$ Given the prospect of ongoing complications from treatment-related toxicities across the illness trajectory, patient-reported outcomes have been recognized as an important endpoint in the management of MM. ${ }^{11,15,16}$

Despite awareness of the importance of patient outcomes in the management of MM, research has largely focused on the physical effects of MM and its treatments; comparatively little is understood about the impact of symptom burden on the psychosocial wellbeing of patients with MM. Research has found that both high symptom burden and mental health symptoms are strong determinants of health-related QoL in patients with MM. ${ }^{17}$ For example, in a recent longitudinal study, Ramsenthaler et al ${ }^{18}$ reported that symptom level, anxiety, and pain were more likely to predict declining 
health-related QoL than demographic or clinical characteristics. However, although studies have examined the impact of symptom burden on health-related QoL, ${ }^{12,14,15}$ limited research has focused on identifying specific symptoms and factors that impact psychosocial wellbeing in patients with MM. Understanding specific factors associated with adverse psychosocial well-being has the potential to guide development of targeted interventions designed to alleviate symptom burden and improve patient QoL.

Furthermore, given the chronic nature of MM, the illness trajectory can be unpredictable, and can result in a perceived sense of loss of control. Perceived control (ie, the capacity to achieve positive and avoid negative outcomes through their own behaviors ${ }^{19}$ has been considered an important modifiable factor in psychosocial well-being. Research among people with different cancer types (eg, early-stage breast cancer) ${ }^{20}$ has found positive associations between perceived control and psychosocial well-being and QoL. However, few studies have examined the impact of perceived control in managing a chronic disease such as MM. Unpredictability of MM can create burden, and given the substantial risk of recurrence ${ }^{10}$ combined with ongoing management of disease and treatment side effects, the potential of a protective factor such as perceived control for psychosocial well-being for patients with MM warrants attention.

As such, the purpose of our study is 2-fold: to identify the most frequently reported areas of distress among individuals living with $\mathrm{MM}$, and to examine how symptom burden and perceived control over the cancer experience are associated with depression, anxiety, and social satisfaction among patients with MM and survivors. To capture the patient experience across the MM illness trajectory, this cross-sectional study included patients with MM regardless of their stage or phase of illness.

\section{Methods}

\section{Procedure}

In 2013, the Cancer Support Community (CSC) launched the Cancer Experience Registry, an online research initiative examining the social and emotional impact of cancer. Individuals diagnosed with MM were invited to answer core registry questions asked of all participants, regardless of their diagnosis, as well as questions specific to living with MM (referred to as the Cancer Experience Registry: Multiple Myeloma). Participants were recruited through CSC and Leukemia \& Lymphoma Society (LLS) community-based affiliates/chapters and online communities, CSC's toll-free support helpline and LLS's information resource center, and other advocacy organizations and social media. Participants were invited to complete the survey online. Although surveys were typically completed at home, in some cases completion occurred in clinic on a computer or tablet. Ethical and Independent Review Services (E\&I) served as the study Institutional Review Board of record. All procedures performed involving human participants were in accordance with the ethical standards of the 1964 Helsinki Declaration and its later amendments or comparable ethical standards. Participants provided electronic informed consent prior to participation.

\section{Participants}

From March 2013 through December 2016, 598 US residents with a history of MM enrolled in the registry; 441 initiated the web-based survey and 289 answered at least 1 question about emotional and social functioning and symptom burden, constituting the current analysis dataset. The racial composition of the analysis sample (89\% white; $6 \%$ black or African American; $2 \%$ other; $3 \%$ preferred not to share) was significantly different $(P=.005)$ from that of nonparticipants $(76 \%$ white; $15 \%$ black or African American; 9\% other), but the samples did not differ on other sociodemographic characteristics or cancer history.

\section{Measures}

Sociodemographic Characteristics and Clinical History Participants provided information about age, sex, race (white, black or African American, or other/multiracial), Hispanic ethnicity, region, annual household income, education (dichotomized as whether the patient graduated from college), and employment status. Participants also reported years since MM diagnosis, history of relapse, history of MM-related kidney disease, MM stage at diagnosis, and number and type of autologous and allogeneic bone marrow transplants. Participants were asked if they were currently receiving treatment of cancer (yes/no) and whether (yes/no) they had ever received chemotherapy, radiation therapy, and biological therapy ("a type of treatment that works with your immune system to help fight cancer or control side effects").

\section{CancerSupportSource}

Participants completed CancerSupportSource, a 25-item distress screening tool that examines physical, social, emotional, and practical concerns and unmet needs. ${ }^{21}$ Participants rated their level of concern $(0=$ not at all; $4=$ very seriously) for each item that began with the stem, "Today, how concerned are you about..." (eg, "Today, how concerned are you about feeling lonely or isolated?" and "Today, how concerned are you about pain and/or physical discomfort?"). Top concerns were assessed descriptively by calculating the proportion of respondents who reported moderate to very serious 
concern for each item. CancerSupportSource data were collected to describe distress and unmet needs, and not included in multivariate analyses.

\section{PROMIS-29}

Participants completed the Patient-Reported Outcomes Measurement Information System 29 Profile V1.0 (PROMIS29), ${ }^{22}$ a collection of 4 -item subscales assessing participant physical and emotional symptoms and functioning. Participants rated each item with reference to the past 7 days; function/satisfaction scales have no time frame. Higher scores represent more of the construct being measured. Scores are converted to standardized T-scores (mean [SD], 50 [10]); normative reference groups are the US general population. Subscale internal consistency reliability ranged from good to excellent (Cronbach $\alpha=0.84-0.96$ ). Physical function and fatigue subscales were used to examine symptom burden and function; depression, anxiety, and social satisfaction subscales were used to examine psychosocial well-being.

\section{Symptom Interference}

Participants rated the extent to which peripheral neuropathy, bone pain, gastrointestinal toxicity, and infection each interfered with daily life in the past 7 days $(0=$ not at all; $4=$ very much); the average of the 4 ratings was calculated to create a symptom interference score (range, $0-4 ; \alpha=0.61$ ).

\section{Illness Control}

For items assessing perceived control, participants rated their perceived control $(0=$ no control at all; $4=$ complete control) over the physical side effects of MM and its treatment, type of follow-up care received, and control over the course of their MM (ie, whether MM will come back or worsen); the average of the 3 ratings was calculated to create a perceived control score (range, $0-4$; $\alpha=0.63$ ). For preparedness to manage side effects, participants indicated in a single item the extent $(0=$ not at all; $4=$ very much) to which they felt their healthcare team had prepared them to manage side effects of MM treatment.

\section{Analysis}

Data analysis was conducted using Stata, version 14 (StataCorp LLP).

\section{Descriptive Analyses}

Descriptive statistics were calculated for all study variables. When reporting descriptive results, scales were collapsed into dichotomous categories for cancer-related distress $(0=$ not at all, slightly; $1=$ moderately, seriously, very seriously), symptom interference $(0=$ not at all, a little bit, somewhat; 1 = quite a bit, very much), perceived control $(0=$ no control, a little; $1=$ moderate amount, a great deal, complete), and PROMIS scales $(0=\mathrm{T}$-scores $<1$ SD than the national average; $1=\mathrm{T}$-scores $\geq 1 \mathrm{SD}$ ) to facilitate reader interpretation. When used in statistical analysis, original scale responses were retained.

\section{Hierarchical Multiple Regression Analyses}

The dependent variables were PROMIS-29 scores on depression, anxiety, and social satisfaction. Pearson and Spearman correlations and chi-square tests were used to examine bivariate relations between dependent variables and potential covariates/independent variables: sociodemographic (age, sex, education, race, ethnicity, annual household income, employment status, regional location), clinical history (years since diagnosis, history of relapse, MM-related kidney disease, disease stage at diagnosis, stem cell transplant history, currently receiving treatment, and treatment after diagnosis), symptom burden and function (PROMIS-29 physical function, PROMIS-29 fatigue, symptom interference), and illness control variables (perceived control, preparedness to manage side effects). To minimize type I error, these potential covariates were selected for regression models based on a priori hypotheses.

Variables significantly associated with dependent variables in bivariate analyses were included in hierarchical multiple linear regression analyses as follows: step 1, sociodemographic variables; step 2, clinical history variables; step 3, symptom burden and function (symptom interference, fatigue, and physical function); and step 4, illness control (perceived control and preparedness to manage side effects). We performed complete case analysis when fitting regression models.

\section{Results}

\section{Clinical Description of the Sample}

\section{Participant Characteristics}

A total of 289 participants responded to at least 1 item about emotional and social functioning and symptom burden. Sociodemographic characteristics and cancer history are presented in Table 1. More than half (56\%) received 1 to 2 autologous transplants, $10 \%$ received $\geq 3$ autologous transplants, and $4 \%$ received allogeneic and autologous transplants; $30 \%$ had not received a stem cell transplant. Most (85\%) received chemotherapy, and $73 \%$ reported currently receiving treatment; $39 \%$ experienced a relapse of MM and $13 \%$ indicated they had MM-related kidney disease.

\section{Cancer-Related Distress}

The most strongly endorsed concerns (moderate to very serious) among patients with MM were related to physical function and symptom burden: eating and 


\section{Table 1. Patient Characteristics (N=289)}

\begin{tabular}{|c|c|}
\hline Characteristic & n (\%) \\
\hline Age, mean (SD), y $(n=249)$ & $62.6(9.0)$ \\
\hline Women $(n=283)$ & $153(54 \%)$ \\
\hline \multicolumn{2}{|l|}{ Race $(n=283)$} \\
\hline Black or African American & $18(6 \%)$ \\
\hline White & $253(89 \%)$ \\
\hline Other & $5(2 \%)$ \\
\hline Prefer not to share & $7(3 \%)$ \\
\hline Hispanic or Latino ethnicity $(n=283)$ & $6(2 \%)$ \\
\hline \multicolumn{2}{|l|}{ Region $(n=263)$} \\
\hline Rural & $66(25 \%)$ \\
\hline Suburban & $142(54 \%)$ \\
\hline Urban & $53(20 \%)$ \\
\hline Do not know & $2(1 \%)$ \\
\hline \multicolumn{2}{|l|}{ Education $(n=280)$} \\
\hline No college & $33(12 \%)$ \\
\hline Some college & $57(20 \%)$ \\
\hline College degree & $114(41 \%)$ \\
\hline Graduate degree & $76(27 \%)$ \\
\hline \multicolumn{2}{|l|}{ Annual household income, \$USD $(n=241)$} \\
\hline$<\$ 20,000$ & $19(8 \%)$ \\
\hline$\$ 20,000-\$ 39,999$ & $43(18 \%)$ \\
\hline$\$ 40,000-\$ 59,999$ & $37(16 \%)$ \\
\hline$\$ 60,000-\$ 79,999$ & $22(9 \%)$ \\
\hline$\$ 80,000-\$ 99,999$ & $20(8 \%)$ \\
\hline$\geq \$ 100,000$ & $32(13 \%)$ \\
\hline Don't know/prefer not to share & $68(28 \%)$ \\
\hline \multicolumn{2}{|l|}{ Employment status $(n=274)$} \\
\hline Full-time & 49 (18\%) \\
\hline Part-time & $12(4 \%)$ \\
\hline Not employed, retired & $127(46 \%)$ \\
\hline Not employed, disability & $68(25 \%)$ \\
\hline Not employed, other & $18(7 \%)$ \\
\hline Years since MM diagnosis, mean (SD) $(n=283)$ & $4.4(4.0)$ \\
\hline Relapse history $(n=259)$ & $102(39 \%)$ \\
\hline MM-related kidney disease $(n=286)$ & $36(13 \%)$ \\
\hline \multicolumn{2}{|l|}{ MM stage at diagnosis $(n=285)$} \\
\hline Stage I & $51(18 \%)$ \\
\hline Stage II & $50(18 \%)$ \\
\hline Stage III & $92(32 \%)$ \\
\hline Other/Do not know & $92(32 \%)$ \\
\hline \multicolumn{2}{|l|}{ Stem cell transplant history $(n=278)$} \\
\hline None & $84(30 \%)$ \\
\hline $1-2$ autologous & $154(56 \%)$ \\
\hline$\geq 3$ autologous & $28(10 \%)$ \\
\hline Allogeneic + autologous & $12(4 \%)$ \\
\hline
\end{tabular}

(continued)

\section{Table 1. Patient Characteristics ( $N=289)$ (cont.)}

\begin{tabular}{|lr|}
\hline Characteristic & $\mathbf{n}(\%)$ \\
\hline Currently receiving treatment $(\mathrm{n}=285)$ & $209(73 \%)$ \\
\hline Treatment after diagnosis & $245(85 \%)$ \\
\hline Chemotherapy & $89(31 \%)$ \\
\hline Radiation therapy & $194(67 \%)$ \\
\hline Biological therapy
\end{tabular}

Abbreviation: MM, multiple myeloma.

nutrition (61\%), physical activity (59\%), moving around (56\%), fatigue (55\%), pain (52\%), and sleep (46\%) (Table 2). Many participants also reported concern about emotional and interpersonal matters (eg, 43\% reported worry about the future and what lies ahead, and $32 \%$ reported feeling sad or depressed).

\section{Symptom Burden and Function}

Several respondents reported that the following types of symptoms interfered with daily life quite a bit or very much: bone pain $(22 \%)$, peripheral neuropathy $(21 \%)$, gastrointestinal toxicity (16\%), and infection (3\%). A total of $38 \%$ of respondents reported physical functioning in ranges suggestive of substantial impairment $(\geq 1$ SD than the national average), and $33 \%$ reported fatigue in ranges suggestive of impairment.

\section{Illness Control}

Only $27 \%$ of participants believed they had control (moderate, a great deal, or complete) over the course of their MM (ie, whether it will come back, get worse, or they will develop a different type of cancer); $48 \%$ perceived having control over the physical side effects of MM; and $88 \%$ over the type of follow-up care they receive. A total of $57 \%$ of participants reported feeling prepared by their healthcare team to manage the side effects of MM treatment.

\section{Psychosocial Well-Being}

Substantial proportions of patients with MM reported psychosocial well-being in ranges suggestive of impairment ( $\geq 1$ SD than the national average) with respect to depression (17\%), anxiety (20\%), and social satisfaction $(29 \%)$.

\section{Correlation and Regression Analyses}

Bivariate associations between independent and dependent variables are presented in Table 3 , and results from hierarchical multiple linear regression analyses are presented in Table 4. All models were significant at all steps; the greatest proportion of variance in each model was accounted for by symptom burden and physical function variables. 


\begin{tabular}{|lc|}
\hline $\begin{array}{l}\text { Table 2. Most Strongly Endorsed Concerns } \\
\text { (N=283) }\end{array}$ \\
\hline
\end{tabular}

Sample sizes ranged from 281 to 287 .

aparticipants indicating "moderately" to "very seriously" concerned.

\section{Depression}

Nine predictors explained $36 \%$ of the variance in depression (adjusted $R^{2}=0.36, F(10,209)=13.10 ; P<.001$ ); in the final model, younger age (semipartial $r=-0.11$; $P=.048$ ), greater symptom interference (semipartial $r=0.11 ; P=.050$ ), greater fatigue (semipartial $r=0.30$; $P<.001$ ), lower perceived control over MM side effects (semipartial $r=-0.16 ; P=.005$ ), and lower preparedness to manage side effects (semipartial $r=-0.11$; $P=.037$ ) were associated with significantly greater depression. Lower education and history of MM-related kidney disease were associated with greater depression at steps 1 and 2 of the model, but became nonsignificant after the addition of symptom burden variables.

\section{Anxiety}

Eight predictors explained $33 \%$ of the variance in anxiety (adjusted $\left.R^{2}=0.33, F(8,206)=13.99 ; P<.001\right)$; in the final model, younger age (semipartial $r=-0.13,-0.023$ ), greater symptom interference (semipartial $r=0.11 ; P=.046$ ), greater fatigue (semipartial $r=0.32 ; P<.001$ ), and lower perceived control over MM side effects (semipartial $r=-0.17 ; P=.002$ ) were associated with greater anxiety. Women reported significantly greater anxiety at steps 1 and 2 of the model, but sex became nonsignificant after the addition of symptom burden variables.

\section{Social Satisfaction}

Eight predictors explained $62 \%$ of the variance in social satisfaction (adjusted $R^{2}=0.62, F(9,207)=40.31 ; P<.001$ ); in the final model, poorer physical function (semipartial $r=0.28 ; P<.001$ ) and greater fatigue (semipartial $r=-0.31 ; P<.001)$ were associated with lower social satisfaction. Lower education, history of relapse, and ever receiving chemotherapy were associated with lower social satisfaction at steps 1 and 2 of the model, but became nonsignificant after the addition of symptom burden variables.

To summarize, results from hierarchical multiple linear regression models predicting depression, anxiety, and social satisfaction indicate that higher symptom burden and lower perceived control are strongly associated with poorer QoL among patients with MM.

\section{Discussion}

Advances in the treatment of MM have extended the life expectancy of patients ${ }^{1,4,7}$; however, as patients with MM live longer, they also spend more time receiving treatments that can be physically and mentally demanding. ${ }^{15}$ As such, this study explored the physical and psychosocial experiences of illness of patients with MM. Our results highlight 3 primary findings. First, we provide a robust description of distress and unmet needs that impact patients with MM in their daily lives. We also demonstrate that many patients with MM not only report symptom burden and interference with daily functioning but also perceive a lack of control over their lived experiences. And finally, our results highlight the specific contributions of physical factors, such as symptom burden, and psychosocial factors, such as perceived control, on patients' overall psychosocial well-being. Taken together, these results highlight considerable distress, lack of control, and frequent symptom burden and interference among many patients with MM. Furthermore, the findings present opportunities to develop targeted interventions that may improve the lives of patients with MM. 


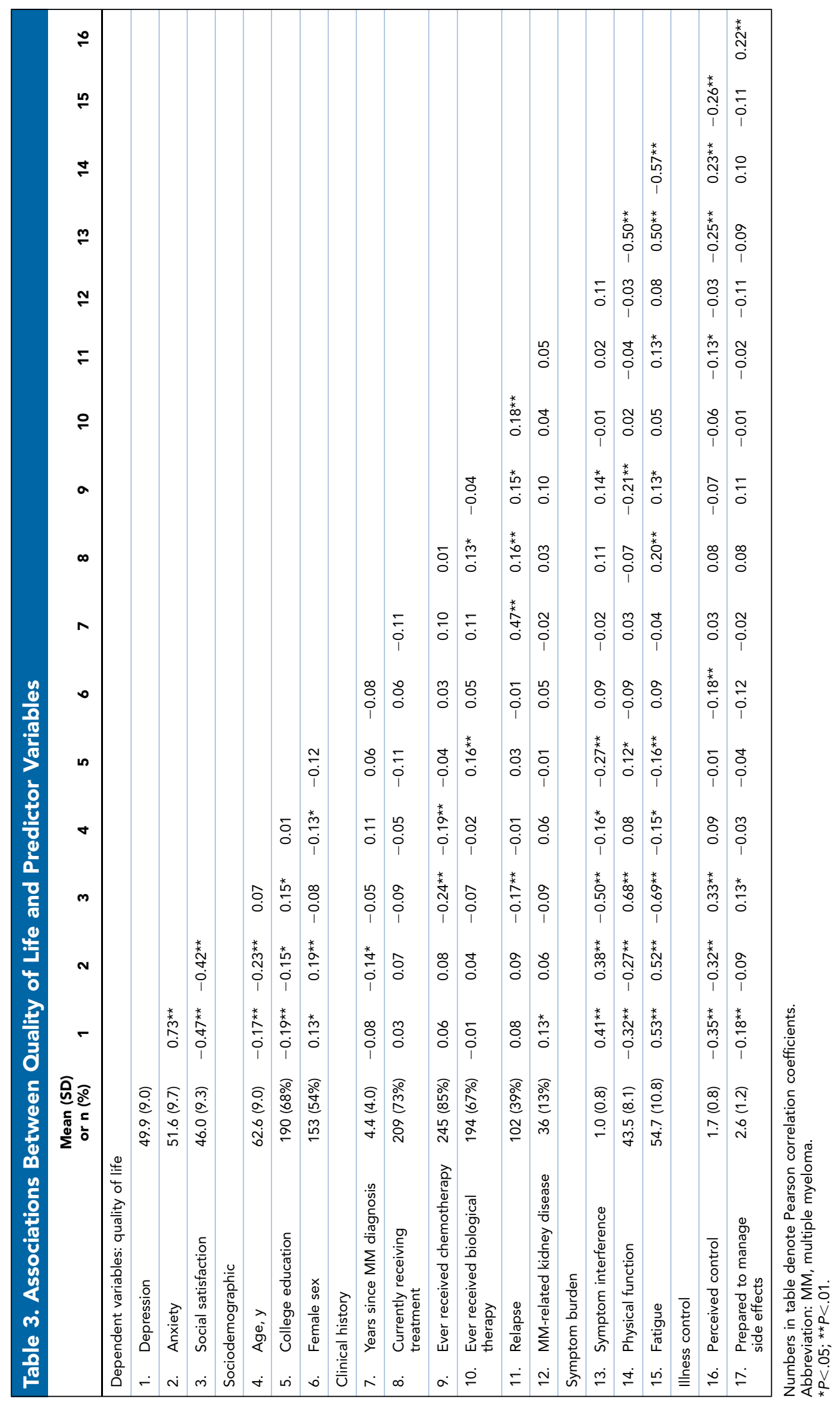


Table 4. Hierarchical Multiple Regression Analyses Predicting Depression, Anxiety, and Social Satisfaction

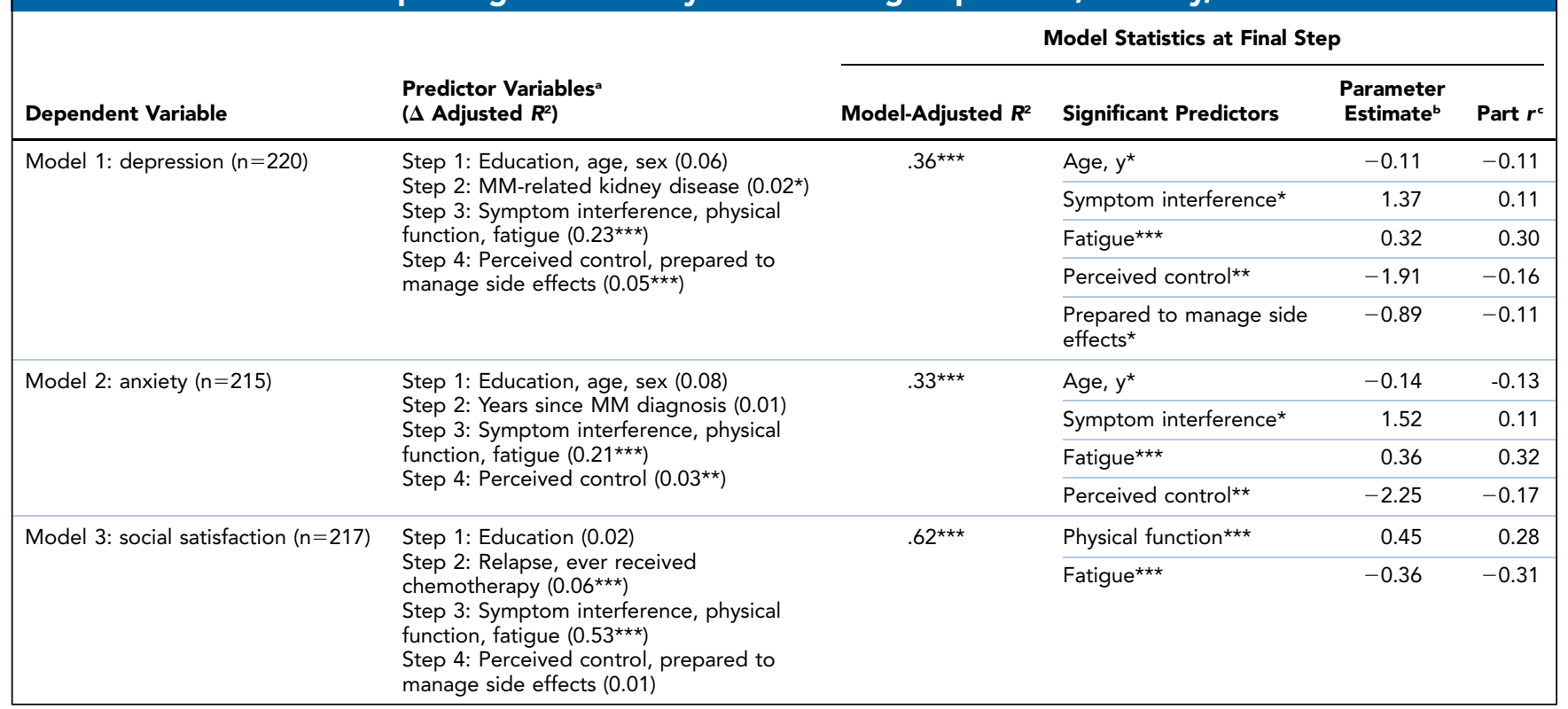

Abbreviation: MM, multiple myeloma.

avariables significantly associated with response variable in bivariate analysis.

bThe parameter estimates ( $\beta$ coefficients) are the average change in the response variable associated with a unit change in the predictor, assuming other predictors are held constant.

'Semipartial correlation.

${ }^{\star} P<.05 ;{ }^{\star \star} P<.01 ;{ }^{\star \star \star} P<.001$

Consistent with prior research, ${ }^{15,23}$ we found that patients with MM commonly reported concerns and cancer-related distress related to symptoms and side effects. For example, $>50 \%$ were distressed by fatigue, pain, and limited physical function, and nearly $50 \%$ were distressed by sleep difficulties. In contrast to prior studies, ${ }^{15,23}$ we also examined concerns about eating and nutrition, and found, notably, that this was the greatest concern in the current sample, with $61 \%$ of respondents reporting being moderately to very concerned about eating and nutrition. Unfortunately, interventions for these symptoms often present a challenge. Appetite stimulants yield marginal results, and most pharmacologic approaches to cancer cachexia do not yield improvements in lean body mass or physical function. ${ }^{24-26}$ Similarly, fatigue management requires multimodal intervention, and many patients do not have access to evidence-based approaches because of cost and limited insurance coverage. ${ }^{27,28}$ Regarding sleep difficulties, although sometimes managed in the short term with medications, they are best addressed using behavioral interventions, such as cognitive behavioral therapy. ${ }^{29,30}$ These concerns, which have meaningful life impact, are unlikely to be addressed adequately through standard cancer care pathways. Our findings suggest the need for more targeted attention and intervention related to appetite and nutrition, fatigue management, and physical function in patients with MM.
Building upon these results, we also found that patients with MM perceived a lack of control over their lived experiences of illness, which had a small but significant association with greater depression and anxiety, even after controlling for the role of physical symptom burden. This is concerning, and perhaps reflective of inadequate attention to symptom assessment and management in routine cancer care. Studies of early integrated palliative care during active cancer treatment show that attending to symptoms improves patients' overall $\mathrm{QoL}^{31-33}$ and may lead to prolonged survival. ${ }^{34}$ Our findings suggest that routine cancer care for $\mathrm{MM}$ does not address distress adequately, and that targeted efforts to improve symptom management have the potential to yield improved illness experiences for patients with MM. However, research has shown that palliative care services are underutilized among patients with hematologic malignancies, and most trials of integrated palliative care have excluded hematology patients. ${ }^{35}$ Thus, efforts are needed to facilitate the early integration of palliative care as part of MM management. In addition, assessing symptom burden and QoL outcomes throughout the illness trajectory as part of routine care has the potential to identify areas of concern accurately, and enable tailoring of services, care, and interventions to meet patient needs effectively.

Last, we found that physical function and symptom burden among patients with MM are associated with 
depression, anxiety, and social satisfaction. Although these constructs are complex, the pattern is consistent and clear. As such, these analyses support the notion that targeted attention to symptom burden and physical function of patients with MM has the potential to yield dividends in improving patients' overall lived experiences of MM. More research is needed in this area, along with the development of targeted interventions specifically for patients with MM.

Our study has limitations. Clinical history was provided by participants and we did not ask about specific therapeutic agents; given the complexity of MM, selfreported medical history (including relapse, history of MM-related kidney disease, and disease stage) may not be fully accurate, and we are not able to differentiate between active versus maintenance therapy. In addition, we do not have data on nonparticipants; patients with MM who participate in online research may differ from the overall population of patients with MM and survivors. In this instance, we believe it is of note that the physical, emotional, and practical burden of living with MM remains substantive even in a sample that may overrepresent higher-functioning patients. Furthermore, structural barriers, including limited access to the internet or restricted time for completion, may select more socioeconomically advantaged patients with $\mathrm{MM}$ into our sample. In this study, we were able to compare those who began the website-based survey but stopped prematurely and those who completed most or all of the survey, including items pertinent to the present study. Although we did not find differences in many sociodemographic characteristics and clinical variables, we did observe a significant difference in racial distribution. This suggests there may be underrepresentation of underserved groups. The study was cross-sectional in nature and included a heterogenous population comprising patients with MM receiving treatment, long-term survivors, and patients with newly diagnosed MM. Thus, our analyses can describe associations but cannot attribute causation.

\section{Conclusions}

Patients with MM experience substantive concerns about the physical, emotional, and practical impact of disease. Symptom burden significantly predicted poorer QoL outcomes, including depression, anxiety, and social satisfaction. Moreover, perceived lack of control over illness was associated with greater anxiety and depression among our national sample of patients with MM. As long-term survival for patients with MM improves, the need to address symptom burden, integrate palliative care, and enhance social and emotional support becomes ever more important.

Submitted June 3, 2019; accepted for publication March 19, 2020

Author contributions: Study concept: LeBlanc, Cole, Buzaglo. Funding acquisition: Buzaglo. Methodology: Zaleta, Miller, Buzaglo. Supervision: Zaleta, Buzaglo. Data curation: Zaleta, Miller. Investigation: Buzaglo. Formal analysis: Zaleta, Miller, Olson, McManus. Writing-original draft: All authors.

Disclosures: Dr. Zaleta, Dr. Miller, Dr. Olson, Dr. Yuen, Ms. McManus disclose that their institution has received grant/research support from Amgen Oncology, Bristol Myers Squibb, Celgene Corporation, Genentech, Takeda Oncology, and Novartis during the conduct of the study. Dr. LeBlanc has disclosed that he has received honoraria from AbbVie, Agios, AstraZeneca, Amgen, Carevive, Celgene, Daiichi-Sankyo, Flatiron, Helsinn, Heron, Medtronic, Otsuka, Pfizer, Seattle Genetics, UpToDate, and Welvie, and has received grant/research support from AstraZeneca and Seattle Genetics outside the submitted work. Dr. Cole has disclosed that he has not received any financial consideration from any person or organization to support the preparation, analysis, results, or discussion of this article. Dr. Buzaglo discloses that her institution received grants from Amgen Oncology, Bristol Myers Squibb, Celgene Corporation, Genentech, Takeda Oncology, and Novartis during the conduct of the study; has received honoraria from Carevive; and is currently employed by Concerto HealthAl.

Funding: This work was supported by funding from Genentech (IDOEVIAG3393), Novartis Pharmaceuticals Corporation (IDOEEJAG3394), Celgene (IDOETJAG3395), Amgen (IDOECKAG3396), Takeda Oncology (ID0ERKAG3397), and Bristol-Myers Squibb (IDOEALAG3398).

Correspondence: Alexandra K. Zaleta, PhD, Cancer Support Community, Research and Training Institute, 520 Walnut Street, Suite 1170, Philadelphia, PA 19106. Email: azaleta@cancersupportcommunity.org

\section{References}

1. Siegel RL, Miller KD, Jemal A. Cancer statistics, 2019. CA Cancer J Clin 2019:69:7-34

2. Kumar SK, Dispenzieri A, Lacy MQ, et al. Continued improvement in survival in multiple myeloma: changes in early mortality and outcomes in older patients. Leukemia 2014;28:1122-1128.

3. Cook G, Ashcroft AJ, Cairns DA, et al. The effect of salvage autologous stemcell transplantation on overall survival in patients with relapsed multiple myeloma (final results from BSBMT/UKMF Myeloma X Relapse [Intensive]): a randomised, open-label, phase 3 trial. Lancet Haematol 2016;3:e340-351.

4. Ríos-Tamayo R, Sánchez MJ, Puerta JM, et al. Trends in survival of multiple myeloma: a thirty-year population-based study in a single institution. Cancer Epidemiol 2015;39:693-699.

5. Fonseca $\mathrm{R}$, Abouzaid S, Bonafede $\mathrm{M}$, et al. Trends in overall survival and costs of multiple myeloma, 2000-2014. Leukemia 2017;31:1915-1921.

6. Kyle RA, Rajkumar SV. Criteria for diagnosis, staging, risk stratification and response assessment of multiple myeloma. Leukemia 2009;23:3-9.

7. Laubach JP, Mitsiades CS, Mahindra A, et al. Novel therapies in the treatment of multiple myeloma. J Natl Compr Canc Netw 2009;7: 947-960.
8. Joks M, Jurczyszyn A, Machaczka M, et al. The roles of consolidation and maintenance therapy with novel agents after autologous stem cell transplantation in patients with multiple myeloma. Eur J Haematol 2015; 94:109-114.

9. Tacchetti P, Terragna C, Galli M, et al. Bortezomib- and thalidomideinduced peripheral neuropathy in multiple myeloma: clinical and molecular analyses of a phase 3 study. Am J Hematol 2014;89: 1085-1091.

10. Rajkumar SV. Updated diagnostic criteria and staging system for multiple myeloma. Am Soc Clin Oncol Educ Book 2016;35:e418-423.

11. Ramsenthaler C, Osborne TR, Gao W, et al. The impact of disease-related symptoms and palliative care concerns on health-related quality of life in multiple myeloma: a multi-centre study. BMC Cancer 2016;16:427.

12. Mols F, Oerlemans $\mathrm{S}$, Vos $A H$, et al. Health-related quality of life and disease-specific complaints among multiple myeloma patients up to $10 \mathrm{yr}$ after diagnosis: results from a population-based study using the PROFILES registry. Eur J Haematol 2012;89:311-319.

13. de Wet $R$, Lane $H$, Tandon $A$, et al. 'It is a journey of discovery': living with myeloma. Support Care Cancer 2019;27:2435-2442. 
14. Chakraborty R, Hamilton BK, Hashmi SK, et al. Health-related quality of life after autologous stem cell transplantation for multiple myeloma. Biol Blood Marrow Transplant 2018;24:1546-1553.

15. Boland E, Eiser C, Ezaydi Y, et al. Living with advanced but stable multiple myeloma: a study of the symptom burden and cumulative effects of disease and intensive (hematopoietic stem cell transplant-based) treatment on health-related quality of life. J Pain Symptom Manage 2013;46:671-680.

16. Kvam AK, Waage A. Health-related quality of life in patients with multiple myeloma-does it matter? Haematologica 2015;100:704-705.

17. Jordan K, Proskorovsky I, Lewis $\mathrm{P}$, et al. Effect of general symptom level, specific adverse events, treatment patterns, and patient characteristics on health-related quality of life in patients with multiple myeloma: results of a European, multicenter cohort study. Support Care Cancer 2014;22: 417-426.

18. Ramsenthaler C, Gao W, Siegert RJ, et al. Symptoms and anxiety predict declining health-related quality of life in multiple myeloma: a prospective, multi-centre longitudinal study. Palliat Med 2019;33:541-551.

19. Wallston KA, Wallston BS, Smith $S$, et al. Perceived control and health. Curr Psychol Res Rev 1987;6:5-25.

20. Bárez M, Blasco T, Fernández-Castro J, et al. Perceived control and psychological distress in women with breast cancer: a longitudinal study. J Behav Med 2009;32:187-196.

21. Buzaglo JS, Zaleta AK, McManus $S$, et al. CancerSupportSource ${ }^{\circledR}$ : validation of a revised multi-dimensional distress screening program for cancer patients and survivors. Support Care Cancer 2020;28:55-64.

22. Cella D, Riley W, Stone A, et al. The Patient-Reported Outcomes Measurement Information System (PROMIS) developed and tested its first wave of adult self-reported health outcome item banks: 2005-2008. J Clin Epidemiol 2010;63:1179-1194.

23. Ramsenthaler C, Kane P, Gao W, et al. Prevalence of symptoms in patients with multiple myeloma: a systematic review and meta-analysis. Eur J Haematol 2016;97:416-429.

24. Khatib MN, Gaidhane A, Gaidhane S, et al. Ghrelin as a promising therapeutic option for cancer cachexia. Cell Physiol Biochem 2018;48:2172-2188.
25. Suzuki H, Asakawa A, Amitani H, et al. Cancer cachexia-pathophysiology and management. J Gastroenterol 2013;48:574-594.

26. Yennurajalingam S, Bruera E. Role of corticosteroids for fatigue in advanced incurable cancer: is it a 'wonder drug' or 'deal with the devil'. Curr Opin Support Palliat Care 2014;8:346-351.

27. Basen-Engquist K, Alfano CM, Maitin-Shepard M, et al. Moving Research Into Practice: Physical Activity, Nutrition, and Weight Management for Cancer Patients and Survivors. NAM Perspectives. Washington, DC: National Academy of Medicine; 2018.

28. Groeneveldt L, Mein G, Garrod R, et al. A mixed exercise training programme is feasible and safe and may improve quality of life and muscle strength in multiple myeloma survivors. BMC Cancer 2013;13:31.

29. Jacobs GD, Pace-Schott EF, Stickgold R, et al. Cognitive behavior therapy and pharmacotherapy for insomnia: a randomized controlled trial and direct comparison. Arch Intern Med 2004 164:1888-1896.

30. Sivertsen B, Omvik S, Pallesen S, et al. Cognitive behavioral therapy vs zopiclone for treatment of chronic primary insomnia in older adults: a randomized controlled trial. JAMA 2006;295:2851-2858.

31. Hearn J, Higginson IJ. Do specialist palliative care teams improve outcomes for cancer patients? A systematic literature review. Palliat Med 1998;12:317-332.

32. Jacobsen J, Jackson V, Dahlin C, et al. Components of early outpatient palliative care consultation in patients with metastatic nonsmall cell lung cancer. J Palliat Med 2011;14:459-464.

33. Greer JA, Jackson VA, Meier DE, et al. Early integration of palliative care services with standard oncology care for patients with advanced cancer. CA Cancer J Clin 2013;63:349-363.

34. Mayor S. Electronic self reporting of symptoms may improve survival in patients with metastatic cancer. BMJ 2017;357:j2724.

35. LeBlanc TW, O'Donnell JD, Crowley-Matoka M, et al. Perceptions of palliative care among hematologic malignancy specialists: a mixedmethods study. J Oncol Pract 2015;11:e230-238. 